\title{
Comparer, mesurer, classer
}

L'art périlleux de la leximétrie du licenciement

Comparing, measuring, ranking: the perilous art of leximetrics applied to dismissal procedures

\section{Raphaël Dalmasso et Thierry Kirat}

\section{OpenEdition \\ Journals}

Édition électronique

URL : http://journals.openedition.org/travailemploi/3421

DOI : 10.4000/travailemploi.3421

ISSN : $1775-416 \mathrm{X}$

Éditeur

DARES - Ministère du Travail

Édition imprimée

Date de publication : 15 décembre 2009

Pagination : 33-46

ISSN : 0224-4365

Référence électronique

Raphaël Dalmasso et Thierry Kirat, « Comparer, mesurer, classer », Travail et Emploi [En ligne],

120 | octobre-décembre 2009, mis en ligne le 30 décembre 2011, consulté le 30 avril 2019. URL :

http://journals.openedition.org/travailemploi/3421; DOI : 10.4000/travailemploi.3421 


\title{
Comparer, mesurer, classer: l'art périlleux de la leximétrie du licenciement
}

\author{
Raphaël Dalmasso $\left({ }^{*}\right)$, Thierry Kirat $(* *)$
}

\begin{abstract}
L'article discute des indicateurs de protection de l'emploi, sous un angle assez peu présent dans les travaux existants: en recourant à la méthode des équivalents fonctionnels en droit comparé, l'analyse du droit du licenciement dans différents pays est menée en partant des situations et des procédures plutôt que des règles visant directement les conditions de la rupture du contrat de travail. La méthode permet de saisir certaines règles procédurales qui ne sont pas, dans les pays de Common law, imposées directement par une réglementation expresse. Nous soutiendrons que des mesures de la réglementation sous la forme d'indicateurs ne sont pas des outils de comparaison, mais de classement: l'ordinal et comparatif forment deux perspectives très différentes et irréductibles.
\end{abstract}

Les indicateurs de protection de l'emploi de l'OCDE (législation de protection de l'emploi (LPE)) et du programme Doing Business de la Banque mondiale et de la Société financière internationale (indicateur Employing Workers $(E W)$ ) sont parmi les données empiriques les plus couramment utilisées dans les travaux d'évaluation économique appliquée des effets de la réglementation sur les performances du marché du travail. S'ils sont différents dans leur contenu et dans la méthodologie de leur construction (KIRAT, 2006), les indicateurs de l'OCDE et de Doing Business (DB) partagent l'objectif d'exprimer sous la forme d'indices synthétiques le degré de rigueur et de formalisme des règles applicables dans divers États en matière de recrutement et de licenciement de salariés. Ces indicateurs sont basés sur une série de variables qualitatives dont la vocation est de capturer de manière uniforme les caractéristiques de la législation de l'emploi en vigueur dans ces pays, de façon à permettre des classements internationaux. Ils relèvent d'une démarche qui a pu être qualifiée de «leximétrique» (SIEMs, 2005) et d'une visée ordinale: la mesure de la réglementation est en effet orientée vers l'établissement de classements internationaux.

Après le travail précurseur de BERTOLA, BOERI et CAZES (2000), la méthode des indicateurs fait depuis les années récentes l'objet d'un regain d'attention sous plusieurs angles: outre des problèmes techniques et statistiques, ils ont été discutés au regard

(*) CERIT-CRDP, Nancy 2, associé au Centre d'études de l'emploi ; raphael.dalmasso@univ-nancy2.fr

(**) CNRS, IRISSO (Paris-Dauphine), associé au Centre d'études de 1'emploi; thierry.kirat@dauphine.fr. Cet article résulte de la réponse à l'appel d'offres de la DARES sur «L'évaluation économique du droit du travail», réalisée sous l'égide de l'Institut international pour les études comparatives». de leur réalisme dans la perception du fonctionnement des normes juridiques (Ahlering, DeAKIN, 2005; SiEMs, 2005), ou du fait qu'ils procèdent d'une lecture quelque peu étriquée et unilatérale de la réglementation et de ses destinataires (ARRUÑADA, 2007). Enfin, le Bureau international du travail (BIT) a mis l'accent sur le fait que les indicateurs $D B$ contiennent des implications économiques et politiques au regard du fait que les réformes du droit du travail susceptibles d'améliorer la situation d'un pays dans le classement international supposerait une rupture des engagements internationaux noués dans le cadre de nombre de conventions de l'Organisation internationale du travail (OIT) (BERG, CAZES, 2007; BIT, 2007). Récemment, Deakin, Lele et Siems ont proposé des indices longitudinaux, alternatifs à ceux de $D B$, afin de mettre à l'épreuve la théorie des Legal Origins pour 1'explication de la dynamique réglementaire en droit du travail et en droit des sociétés (Deakin, Lele, Siems, 2007).

Cet article entend apporter une contribution supplémentaire à la discussion des indicateurs de protection de l'emploi, sous un angle particulier assez peu présent dans les travaux existants : en s'appuyant sur la méthode des équivalents fonctionnels défendue par certains spécialistes reconnus du droit comparé, nous mettrons en discussion les approches du droit du licenciement dans différents pays, en partant non pas des règles visant expressément les conditions de la rupture du contrat de travail, mais des situations et des procédures. Nous entendons par là soutenir que certaines règles procédurales du licenciement ne sont pas, notamment dans les pays de Common law, imposées directement par une réglementation directe de la rupture du contrat de travail, mais sont présentes dans la réalité, dans les entreprises, et échappent largement à la mesure de la réglementation offerte par les indicateurs de 
LPE. Cela illustre à quel point les deux domaines de savoirs, pourtant a priori complémentaires, que sont le droit comparé d'une part et la recherche statistique, économique et économétrique, d'autre part, sont cloisonnés et structurellement étanches l'un à l'autre. Pourtant, comme nous l'argumenterons plus avant, la leximétrie repose sur une théorie implicite du droit comparé et du fonctionnement des règles de droit: elle implique une mise en équivalence généralisée de normes légales imparfaitement comprises et mesurées plutôt que la recherche des équivalents fonctionnels. Par conséquent, nous soutiendrons que des mesures de la réglementation sous la forme d'indicateurs ne sont pas des outils de comparaison, mais de classement: l'ordinal et comparatif forment deux perspectives très différentes et irréductibles.

La première partie dressera le cadre d'analyse de la comparaison des modalités de rupture du contrat de travail dans différents pays en mettant au premier plan le problème de la comparaison des procédures, plus précisément des cheminements procéduraux de la rupture du contrat de travail en vigueur dans ce que nous appellerons des «ordonnancements juridiques» particuliers. S'appuyant sur la section précédente, la deuxième partie s'attachera plus directement à la discussion du contenu informationnel des indicateurs de LPE. Elle insistera sur les «angles morts» qui les caractérisent, notamment la mise en œuvre de la réglementation dans le contentieux et la jurisprudence, voire dans des dispositions conventionnelles.

\section{Comparer les procédures}

Il suffit de consulter la valeur des indicateurs, qu'il s'agisse de la LPE ou de $E W$, pour comprendre qu'une association univoque y est faite entre l'appartenance à telle ou telle famille de droit et la propension à réglementer la relation d'emploi en général, la rupture du contrat de travail en particulier. Les pays appartenant au monde de la Common law seraient peu portés à l'interférence de la puissance publique dans les relations contractuelles de travail, alors qu'à l'inverse ceux qui appartiennent à la famille du droit civil auraient une (fâcheuse) tendance à réglementer et à imposer des contraintes procédurales fortes, vecteurs de coûts importants, à la pratique du licenciement, individuel ou collectif, pour motif personnel ou pour motif économique.

Ces jugements méritent que l'on s'y attarde. Nous les reconsidérerons d'abord sous la perspective du droit comparé en général, puis au regard d'une analyse juridique empirique des procédures de licenciement dans plusieurs «ordonnancements juridiques $»$.

\section{Apports d'une perspective de droit comparé}

Comme nous l'étayerons dans la deuxième partie, les indicateurs de LPE mesurent moins le droit du travail sous l'angle de sa mise en œuvre que sous celui des normes légales, c'est-à-dire des énoncés normatifs formulés dans les textes législatifs et réglementaires. Le problème de l'objet de la comparaison est la question centrale du droit comparé depuis son acte de naissance au début du $\mathrm{XX}^{\mathrm{e}}$ siècle, et fait l'objet, sous la plume de spécialistes reconnus, d'une formulation renouvelée.

\section{Législation comparée vs jurisprudence comparative}

L'un des plus importants juristes comparatistes du début du $\mathrm{XX}^{\mathrm{e}}$ siècle, Edouard Lambert, mettait les auteurs d'analyses comparées en garde contre les biais et les risques d'interprétations erronées induits par le fait de ne considérer que les seuls textes législatifs. Ce procédé a pour effet d'éloigner le commentateur de l'observation de la mise en œuvre du droit légiféré. La mise en garde de Lambert, formulée dans son Gouvernement des juges publié en 1921, portait plus particulièrement sur la comparaison des systèmes de Common law et de ceux issus de la tradition romano-germanique. S'agissant des États-Unis, Lambert montrait la portée de l'erreur de méthode que véhicule le fait de réduire le droit en vigueur aux normes décrites dans la législation, plus précisément dans la Statute law: «[...] le statut américain n'offre au juriste, qui cherche à se renseigner sur le droit effectivement appliqué aux États-Unis, qu'un instrument fort insuffisant et qui, manié isolément, risquerait dans un grand nombre de cas de le conduire à des conclusions absolument erronées » (LAMBERT, 1921, pp. 254-255).

Or, c'était précisément dans ce travers qu'étaient tombés les organes de droit comparé consacrés à la diffusion internationale des législations étrangères, dont les Annuaires de législation étrangère et de législation française publiés par la Société de législation comparée. La connaissance scientifique des droits étrangers exige alors, selon Lambert, de situer les législations étrangères «[...] dans l'encadrement d'institutions nationales qui seul peut permettre de voir si elles sont conditionnées ou non par les particularités économiques ou les traditions légales du milieu où elles sont destinées à s'appliquer» (LAMBERT, 1921, p. 239) et, surtout, de comprendre «la forme où elles ont été effectivement poursuivies dans la pratique» (ibidem).

La comparaison des législations nationales devait céder le pas à une autre méthode, plus ouverte à la vie du droit et à son expérience pratique, qui se révèle devant les tribunaux, ouvrant ainsi une voie à une lecture contentieuse de la dynamique du droit. La proposition de Lambert de mettre en œuvre une méthode de «jurisprudence comparative» n'a pas eu le succès et la prospérité qu'elle aurait dû recevoir, à tel point que la discipline du droit comparé est à 
la fois sous-pratiquée dans le monde de la recherche juridique, et marquée par une tendance à la superposition de présentations de droits positifs nationaux dans tel ou tel domaine. Dans une période plus actuelle, des juristes comparatistes se sont employés à reformuler les principes de méthode fondamentaux de la discipline du droit comparé : il s'agit moins de comparer terme à terme des normes de droit positif (ou de contraster leur existence dans un pays, leur absence dans un autre) que de reconstituer des équivalents fonctionnels (ZwEIGERT, Kötz, 1998) ou de dépasser les différences de styles judiciaires par une analyse des cas (MARKESINIS, 1997).

\section{Normes de droit positif vs équivalents fonctionnels}

L'ouvrage de ZwEIGERT et KöTZ (1998) constitue à cet égard une référence incontournable. Les propositions méthodologiques formulées par ces auteurs permettent de reconsidérer la question de savoir si la méthode uniforme de construction des variables et des indices élémentaires de protection de l'emploi ne biaise pas les indicateurs synthétiques, notamment lorsqu'on les applique à des systèmes juridiques de tradition différente (civiliste ou de Common law). Il n'est pas intérêt de constater que le projet (très avancé) de juristes de l'université de Cambridge (Royaume-Uni) de construire des indicateurs leximétriques longitudinaux, alternatifs à ceux de l'OCDE et de $D B$, prend appui sur la méthode des équivalents fonctionnels défendue par Zweigert et Kötz. En effet, Deakin, Lele, Siems (2007), retiennent un principe fondamental de l'analyse comparative suggérée par ces derniers, à savoir qu' «un même effet peut résulter de la règle de droit dans un système, et d'instruments d'autoréglementation ou de la législation informelle dans un autre»(1). L'indicateur longitudinal de réglementation du travail de Deakin et al. sur cinq pays (l'Allemagne, les États-Unis, la France, l'Inde, le Royaume-Uni) sur longue période (1970-2006) «prend aussi en compte les règles subsidiaires et les sources normatives non juridiques» (DeAKIN, LeLE, SIEMS, 2007, p. 156).

En effet, Zweigert et Kötz soutiennent que «le principe méthodologique de base du droit comparé est le principe de fonctionnalité» (ZWEIGERT, KöTZ, 1998, p. 35), ce qui constitue le seul procédé capable de prendre en compte le fait que si les systèmes juridiques de toutes les sociétés doivent gérer les mêmes problèmes, ils recourent à des procédés différents. La reconstitution, en partant des fonctions, des méthodes, des procédés, des

(1) La version traduite en français de l'article de Deakin, Lele et Siems n'est pas satisfaisante. La citation en langue anglaise est la suivante: "The same effect might be achieved in one system by a rule of law and in another by self-regulatory instruments or soft law» (p. 144). procédures, pertinentes, est une des tâches fondamentales dans le travail comparatiste. À cet égard, il convient de ne pas retenir une conception restrictive des sources du droit, mais au contraire de traiter comme source «tout ce qui modèle ou affecte le droit vivant dans le système observé, quelle que soit l'attitude des juristes locaux à cet égard», c'est-àdire considérer aussi bien le droit statutaire que le droit coutumier, la jurisprudence et les écrits doctrinaux, les contrats-types et les conditions générales des affaires, les usages et les coutumes des commerçants... (ZwEIGERT, KÖTZ, 1998, pp. 35-36).

À titre d'illustration et en anticipant légèrement sur ce qui suivra, aux États-Unis, la décision de l'employeur de licencier un salarié, quel qu'en soit le motif, est habituellement présentée comme totalement souveraine, sans obligation de motivation et exempte de contraintes procédurales (employment at will). Cette proposition est en partie vraie, et en partie fausse: elle est en partie vraie au regard du fait qu'aucune disposition légale n'impose directement à l'employeur de donner un motif réel et sérieux à la rupture unilatérale du contrat de travail, de donner un préavis au salarié concerné, etc. Mais elle est surtout en partie fausse, compte tenu que certains contrats collectifs peuvent prévoir de tels schémas procéduraux, mais aussi, et surtout, que des dispositions législatives ou règlementaires, voire jurisprudentielles, non directement liées à la relation de travail (par exemple: les droits fondamentaux et la législation antidiscrimination) peuvent avoir pour effet de structurer les conditions dans lesquelles un licenciement doit se dérouler, c'est-à-dire à faire en sorte que certaines procédures soient suivies dans sa réalisation.

En pratique, les indicateurs font la part belle aux procédures qui encadrent les conditions du licenciement pour motif personnel ou pour motif économique et sur les obligations qui pèsent sur les employeurs (durée du préavis, entretien préalable, plan de conversion, autorisation administrative, etc.). Or, si les dispositions expresses de la législation de la plupart des pays de common law sont en nombre plus limité que dans les pays de droit civil et le contenu des règles spéciales de licenciement y est en apparence plus souple, on ne peut pour autant en conclure que le licenciement n'y est pas soumis à des règles. Il existe bien des règles, mais comme elles ne sont pas nécessairement réservées au droit du travail, aucun indicateur ne les prend en considération. Ainsi, dans les pays de common law, les salariés licenciés, en tant que citoyens et justiciables, disposent de droit de contester les conditions de leur licenciement au titre de l'unfair dismissal, équivalent de l'abus de droit du droit français. Les juges, parfois même le législateur, contrôlent le motif du licenciement notamment au regard de la discrimination raciale ou sexuelle, $\mathrm{du}$ harcèlement, et autres droits fondamentaux. Le contrôle de l'unfair dismissal est essentiellement 
judiciaire. Or, le fait que les indicateurs ne prennent pas en compte la jurisprudence conduit à méconnaître des pans entiers de la protection de l'emploi dans les pays de droit anglo-américain. Ces considérations appellent à la plus grande prudence sur la qualité de l'information contenue dans les indicateurs et, partant, quant à la comparabilité des réglementations nationales de protection de l'emploi. C'est à cette prudence que nous invite la comparaison des schémas procéduraux qui entourent la rupture du contrat de travail en France, en Italie et au Royaume-Uni. Il en ressort que l'association des droits des pays de common law avec la flexibilité, et des pays de civil law avec un droit rigide et procédurier, mérite d'être reconsidérée.

\section{Une comparaison des licenciements sous l'angle des procédures}

Nous en venons maintenant à une comparaison exhaustive de schémas procéduraux des licenciements de salariés en contrats «permanents» dans trois pays de l'Union européenne: la France, l'Italie et le Royaume-Uni. Nous ferons, plus ponctuellement, référence au cas des États-Unis.

Le licenciement est le plus souvent décrit comme «un acte par lequel l'employeur rompt unilatéralement le contrat de travail et congédie un ou plusieurs salariés». Dès lors, les juristes décrivent le licenciement comme l'exercice par l'employeur d'un droit de résiliation unilatérale du contrat de travail. C. Camerlinck précise ainsi, évoquant le droit français du licenciement individuel, que, malgré la loi de 1973 relative à la cause réelle et sérieuse de licenciement, «le droit de résiliation subsiste en ce sens que, d'une part, l'employeur n'a aucune autorisation préalable à solliciter [...] et que d'autre part le licenciement irrégulier n'est pas nul, la réintégration ne pouvant être imposée à l'employeur» (CAMERLINCK, 1982, p. 355). Si cette définition est juridiquement exacte, elle ne prend cependant qu'insuffisamment en compte deux aspects contemporains du droit du licenciement.

Tout d'abord, le droit procédural du licenciement produit des contraintes avant et après la rupture des contrats. Ce constat implique, que, dans une comparaison internationale, le schéma temporel du processus de rupture du contrat de travail doit être reconstitué dans sa totalité, sans omettre aucune des étapes qui scandent le processus.

Ensuite, la notion de rupture du contrat doit être redéfinie, et élargie. Dans certaines hypothèses, de simples altérations des contrats de travail conduisent, à terme, à des licenciements. Il convient donc d'intégrer dans l'analyse ces hypothèses nouvelles de rupture à l'initiative de l'employeur.

La méthode retenue dans la recherche qui alimente les réflexions qui suivent vise en premier lieu à aborder dans sa globalité le processus du licen- ciement dans chaque pays étudié, en considérant les procédures mises en place comme des séquences d'action formant une chaîne dont le tribunal est le dernier maillon. La comparaison temporelle procédurale est ainsi des plus larges, prenant en compte toutes les étapes antérieures et postérieures à la rupture.

La comparaison menée redéfinit le champ d'application de ce qu'il est convenu d'appeler une situation de licenciement. Il convient alors d'adopter une conception large des licenciements, intégrant certaines hypothèses de modification et de suspension des contrats menant à la rupture.

Des résultats originaux émergent de l'application de cette méthode comparative aux trois pays étudiés (France, Italie, Royaume-Uni).

\section{Un élargissement procédural temporel du licenciement}

Le droit du licenciement est le plus souvent appréhendé à partir de la rupture du contrat de travail. Le licenciement étant un acte unilatéral de rupture d'un contrat à l'initiative de l'employeur, cette description est a priori exacte. Cette approche traditionnelle, qui fait du licenciement la conséquence de la rupture du contrat, se justifie par le fait que l'acte unilatéral de licenciement n'a d'existence juridique qu'à partir de la notification du congédiement au salarié. Historiquement, cette notification était en outre, dans la plupart des systèmes juridiques, simple, et n'était précédée d'aucune formalité procédurale. En outre, du point de vue de la discussion judiciaire, le litige relatif au licenciement ne peut naître véritablement qu'à partir de la rupture du contrat. Seul cet acte unilatéral produit des effets de droit. Celui-ci peut porter préjudice au salarié et justifier, de sa part, un éventuel recours en justice. Le droit des licenciements est donc, le plus souvent, considéré comme un droit de contestation d'un acte unilatéral, un droit s'opposant à la décision de l'employeur de licencier.

De plus, ce droit, qualifiable d'antidécisionnel, est visible surtout dans sa perspective strictement contentieuse, devant les tribunaux compétents (conseil de prud'hommes en France, Tribunale en Italie, Employment Tribunal au Royaume-Uni, etc.). Inversement, des modes de résolution des litiges liés aux intentions de licenciements, antérieures à sa réalisation et à la saisine du tribunal, apparaissent fréquemment négligés, et demeurent souvent invisibles.

Nous nous proposons de cadrer autrement le schéma temporel résumé ci-dessus.

En premier lieu, il apparaît que les systèmes juridiques étudiés prévoient désormais des normes procédurales antérieures aux licenciements, créant des obligations à la charge de l'employeur, du salarié ou des institutions représentatives du 
personnel avant toute rupture, voire tout projet de rupture. Ces normes tendent à se multiplier et se diversifier. Pour comprendre le droit contemporain du licenciement, il convient de recenser ces étapes antérieures à l'acte de rupture (sur ces procédures dites pro décisionnelles et leur rapport avec les procédures anti décisionnelles, voir DaLmasso (2009, p.111 et ss.).

En second lieu, s'il n'est pas illégitime de considérer le droit du licenciement dans une perspective judiciaire, il convient de savoir que celle-ci est, aujourd'hui, largement incomplète et insatisfaisante. En effet, les procédures de contestation du licenciement se sont largement diversifiées, sous l'impulsion de divers modes alternatifs de règlements des litiges, dans les pays étudiés. Aussi, la perspective uniquement judiciaire du licenciement est-elle devenue, dans le panorama global et complexe de ce droit, trop restreinte.

Les tableaux 1 et 2 , présentés en fin d'article, exposent les étapes procédurales jalonnant, dans les pays étudiés, les processus du licenciement et de sa contestation. Ces tableaux retracent ainsi, d'un point de vue chronologique, l'intégralité des séquences d'actions vécues par l'employeur et le salarié avant et après la rupture du contrat de travail.

Le tableau 1 réunit toutes les hypothèses de licenciements individuels quel que soit leur motif. Certaines procédures sont cependant spécifiques au caractère économique ou personnel des ruptures (obligation d'adaptation valide uniquement pour les licenciements économiques français, procédure de griefs valables uniquement pour les litiges liés à la personne du salarié en droit anglais).

Le tableau 2 recense les étapes procédurales supplémentaires devant être réalisées par l'employeur lors de licenciements collectifs. En particulier, les représentants du personnel jouent dans tous les pays un rôle grandissant.

Pour les deux tableaux, l'exposition chronologique comparée de ces séquences d'action est riche d'enseignements, qui seront détaillés dans la partie relative aux principaux résultats de la comparaison des schémas procéduraux (infra). Il est d'ores et déjà possible d'indiquer que certaines de ces étapes sont partagées par les trois pays, que d'autres sont spécifiques à un ordonnancement, mais toutes, dans le processus du licenciement, ont une logique propre. En outre, les séquençages des procédures montrent à quel point est insatisfaisante, si ce n'est erronée, la comparaison uniquement fondée sur les taux de recours juridictionnel: en Grande-Bretagne, les contestations des motifs de licenciement se déroulent antérieurement à la concrétisation de la rupture, dans le cas de procédures de griefs internes aux entreprises. Elles sont en quelque peu visibles et, surtout, constituent un préalable obligatoire qui conditionne les possibilités d'activation d'autres voies de contestation: Advisory Conciliation and Arbitration Service (ACAS) puis Employment Tribunal.

\section{Un élargissement du concept de licenciement}

Le licenciement étant un acte de rupture du contrat de travail, il faudrait a priori exclure de l'étude les hypothèses de modification ou de suspension du contrat de travail. Il n'en est cependant rien, compte tenu que les droits français et italien du licenciement économique ont établi des liens entre la rupture du contrat pour motif économique et des hypothèses de modification ou de suspension du contrat.

Le droit français indique, via l'article L. 1233-3 du Code du travail, que le licenciement pour motif économique résulte "d'une suppression ou transformation d'emploi, ou d'une modification, refusée par le salarié, d'un élément essentiel du contrat de travail». Le lien direct entre la modification et la rupture du contrat est expressément reconnu, le refus par le salarié de la modification de son contrat provoquant, le plus souvent, son licenciement. La procédure du licenciement économique, envisagée largement, doit donc inclure la phase de proposition de modification du contrat par l'employeur.

Le droit italien permet aux employeurs, en cas de crise conjoncturelle ou structurelle de l'entreprise, de suspendre des contrats de travail pour motif économique et de demander la prise en charge partielle des salariés par une caisse spécifique gérée par l'Istituto Nazionale della Previdenza Sociale (INPS), la Cassa Integrazione Guadagni (caisse d'intégration des salaires). Pour bénéficier de cette caisse, l'employeur doit procéder à la consultation des syndicats (examen conjoint) et solliciter une autorisation administrative (décret de concession). À l'issue de cette procédure, il peut suspendre les contrats de travail de certains salariés. Ceux-ci percevront une indemnité versée par la caisse d'intégration des salaires. La suspension de ces contrats n'est cependant possible que pour une durée limitée (douze ou vingt-quatre mois maximum selon les hypothèses). À l'issue de cette période, les salariés doivent retravailler dans l'entreprise. Si l'employeur est dans l'impossibilité de reprendre les salariés, il peut enclencher la procédure de licenciement pour réduction de personnel. Le droit italien a donc établi un lien direct entre suspension et rupture du contrat pour motif économique.

Ces deux exemples montrent que l'employeur peut, par des chemins procéduraux variés et inattendus, arriver à rompre des contrats de travail pour motif économique. Ces procédures (surtout la procédure italienne) peuvent tout d'abord apparaître longues et fastidieuses. Elles constituent cependant avant tout des voies procédurales inédites de rupture des contrats pour motif économique à la disposition des employeurs. 


\section{Principaux résultats de la comparaison des schémas procéduraux}

L'analyse comparative procédurale met évidence la très grande complexité des droits nationaux en matière de rupture du contrat de travail. Chaque ordonnancement juridique a fait preuve d'une imagination peu commune pour établir des règles encadrant les litiges susceptibles de naître d'un projet ou d'une décision de licenciement. D'ores et déjà, l'analyse des procédures montre que France, Italie et Royaume-Uni présentent des profils bien distincts, chaque système formant un «type» à lui seul. Trois modèles distincts de traitement juridique des ruptures de contrat de travail ont pu être définis. Nous en présentons brièvement les caractéristiques, en distinguant les licenciements individuels (pour motif économique ou inhérent à la personne), et collectifs (économiques).

\section{Licenciements individuels}

Le système du Royaume-Uni (et plus précisément de Grande-Bretagne) est le plus fermement orienté vers la résolution amiable des litiges individuels de travail en dehors des tribunaux.

Ces procédés sont très diversifiés. Tout d'abord, la résolution des litiges est favorisée au plus tôt, dans l'entreprise, dès que l'employeur a un grief à reprocher à un salarié. Une procédure de règlement des litiges sur le lieu de travail (grievance procedure, ou «procédure de griefs»), assez complexe, doit ainsi obligatoirement être suivie. Le licenciement d'un salarié, si licenciement il y a, ne peut intervenir qu'au terme d'un temps assez long, afin que le travailleur puisse apporter des éléments de défense. Après la rupture, des voies de conciliation sont également prévues et favorisées. Les litiges donnant lieu à un contentieux devant les tribunaux compétents sont moins nombreux que dans les deux autres pays étudiés, les étapes de procédure absorbant en quelque sorte les réclamations. On remarquera à cet égard que les indicateurs internationaux regardent avec faveur l'instauration de telles procédures en amont. L'OCDE relevait ainsi que «le fait le plus marquant [...] est sans doute que, dans les pays pour lesquels l'information est disponible, la plupart des conflits du travail sont résolus au moyen de la conciliation. L'accord peut intervenir entre les parties avant même qu'un recours ait été formé, ou bien être passé en cours d'instruction, le différend étant ainsi réglé à l'amiable avant que le tribunal ait rendu un jugement» (OCDE, Perspectives de l'emploi 2004, p. 71). Relayée ensuite par certains économistes, cette observation est présentée comme une cause des excès supposés du contentieux: la France souffrirait d'un "aménagement insuffisant des possibilités de conciliation au sein de l'entreprise » (Cahuc, Kramarz, 2004, pp. 153-154).

Il n'est pas certain cependant que ces étapes préalables sur les lieux de travail constituent une simplification des procédures de licenciement. Pour l'employeur, la procédure de conciliation est obligatoire dès lors qu'il a un grief à exprimer à l'égard du salarié. La procédure elle-même est complexe et comporte plusieurs étapes, dont le non-respect lui fait courir le risque d'une condamnation devant les tribunaux. Du côté du salarié, l'obligation de former des griefs contre son employeur avant de pouvoir saisir le juge peut être l'occasion de l'obliger à fournir des réponses qui pourront être ensuite produites en justice. La multiplication des séquences peut conduire paradoxalement le salarié à contester la décision de l'employeur devant les commissions de conciliation, et plus tard devant les tribunaux. L'affirmation que le droit du licenciement individuel pour motif personnel du Royaume-Uni est peu contraignant est donc infondée du point de vue de la procédure, qui se révèle d'une complexité assez rarement observable ailleurs.

Le cas du Royaume-Uni donne l'opportunité d'apporter des éléments d'information de nature assez proche, sur le cas des États-Unis où des procédures de griefs sont également mises en œuvre dans le monde de l'emploi salarié.

Le cas des procédures de griefs aux États-Unis, qu'il s'agisse d'entreprises du secteur marchand, d'établissements scolaires ou universitaires, analysé par des sociologues du droit à sensibilité néoinstitutionnelle (Edelman, UGGen, ErLanger, 1999) est riche d'enseignements sur le fait qu'une disposition légale générale, dont l'objet n'est pas directement le droit du licenciement, peut conduire les entreprises à mettre en place des procédures destinées à traiter certains litiges nés d'une situation de travail, alors même qu'aucune obligation de droit positif ne les impose. En l'espèce, suite à la promulgation de l'Egal Employment Opportunity Law, dans le cadre des orientations antidiscriminatoires portées par le Civil Rights Act, un «mythe rationnel» s'est développé et répandu aux États-Unis, sous l'influence de professionnels et de consultants en ressources humaines, selon laquelle la mise en place d'une procédure de griefs au sein des entreprises constituait un moyen, pour elles, de se mettre à l'abri de procédures judiciaires susceptibles d'être intentées par des salariés s'estimant victimes de harcèlements ou de discriminations d'ordre racial ou sexuel. Cette croyance, quant à l'effet de mise à l'abri contre une condamnation pour infraction à la législation fédérale antidiscriminatoire de l'institution de procédures de griefs, a été longtemps juridiquement infondée. Cependant, dans les années 1980, les tribunaux fédéraux ont admis que l'existence d'une telle procédure constituait un élément de preuve non négligeable de la volonté des entreprises de se mettre en conformité avec la loi fédérale. De manière plus générale, les travaux d'EDELMAN et al. $(1999,2008)$ consacrés à l'endogénéisation du droit dans les organisations, y compris du fait de croyances ou de «mythes rationnels», montrent 
sur des bases empiriques et statistiques solides que la complexité procédurale peut être le produit, non pas d'une règle visant expressément et directement une situation, mais d'un ensemble de liens sociojuridiques qui prennent corps sur une loi n'ayant aucun rapport direct avec le monde du travail. Or, précisément, les données juridiques alimentant les indicateurs de protection de l'emploi ne tiennent en aucune façon de ces phénomènes d'induction de schémas procéduraux dans la gestion de l'emploi. En droit français, on peut se demander dans quelle mesure la promotion de la négociation relative à gestion prévisionnelle des emplois et des compétences ne repose pas elle aussi, aussi sur le mythe rationnel d'éviter, en cas de licenciement économique, les contestations individuelles judiciaires des ruptures par les salariés.

Comparé au modèle britannique voire au modèle américain, le système français des licenciements individuels apparaît plus simple. Contre tous les stéréotypes, on se limitera à rappeler qu'en France la procédure de rupture d'un contrat à l'initiative de l'employeur se déroule rapidement. Elle comporte deux étapes très peu formelles (convocation à un entretien préalable, entretien), suivies d'une lettre notifiant le licenciement, qui encadrera les termes du litige en cas de saisine du conseil de prud'hommes. Si contestation il y a, l'employeur en sera rapidement informé, et toute la procédure (conciliation puis jugement) se déroulera devant le conseil de prud'hommes, sans qu'il soit nécessaire de recourir à des tiers conciliateurs ou médiateurs dont l'intervention génère délais et coûts supplémentaires. Il en résulte une concentration des litiges en un seul lieu, avec une relative prévisibilité des résultats.

L'Italie représente un modèle intermédiaire. Le licenciement individuel est encore plus facile et rapide qu'en France, car il n'existe pas de procédure d'entretien préalable au licenciement. Cependant, la saisine obligatoire d'une commission de conciliation extrajudiciaire montre que le recours au tribunal compétent n'est qu'une voie ultime de résolution du litige.

\section{Licenciements collectifs}

L'opposition n'est pas aussi marquée pour les licenciements économiques collectifs, qui sont partout encadrés par des procédures. Deux types peuvent être dégagés.

Le premier type regroupe la France et l'Italie, qui multiplient les procédures préalables à tout licenciement collectif (information et consultation sur le plan de sauvegarde de l'emploi, examen conjoint sur la situation de l'entreprise, etc.), voire tout avant-projet de licenciement, avec notamment la procédure de bénéfice de la Cassa Integrazione Guadagni Straordinaria (CIGS) et, en France, la négociation d'accords de méthode sur la procédure de licenciement. On constate donc que la procé- dure de licenciement collectif est en expansion bien avant les ruptures.

Une première lecture de ces étapes procédurales peut conduire à estimer qu'elles constituent, pour l'employeur, des tracasseries supplémentaires, voire inutiles. Cependant, ces dispositions préalables aux licenciements visent aussi à organiser de la manière la plus pacifiée possible les ruptures, suspensions ou modifications collectives de contrats pour motif économique.

La signification de ces nombreuses étapes procédurales supplémentaires est donc ambivalente. Certes, ces procédures retardent et encadrent les ruptures, et imposent aux employeurs une plus grande anticipation. Cependant, elles constituent aussi des voies pour parvenir à rompre en une fois, de manière relativement paisible, une pluralité de contrats.

Le rôle des institutions représentatives du personnel françaises et italiennes (comité d'entreprise et syndicats) est révélateur de cette ambivalence. Celles-ci jouent un rôle décisionnel de plus en plus important. Sans parler de cogestion, car le choix de la restructuration et de rupture des contrats demeure un choix purement patronal, il faut noter la responsabilité grandissante des institutions représentatives du personnel dans ces processus de suspension, modification et rupture collective des contrats. Elles influent ainsi sur les choix de gestion de l'employeur (notamment en matière d'ordre des licenciements ou de reclassement), tout en contribuant à les asseoir. Le développement français des procédures relatives à la gestion prévisionnelle des emplois et des compétences, avant même tout avant projet de licenciement, montre l'attrait opéré par ces mécanismes procéduraux pour les employeurs, puis le législateur.

Le second type renvoie au droit du Royaume-Uni, moins engagé dans ce processus de prévision des licenciements collectifs. Cependant, ce pays est tenu par le droit communautaire, qui a prévu en cas de licenciement collectif une information et une consultation des institutions représentatives du personnel, et ne peut donc pas se situer en opposition complète avec les logiques des deux autres systèmes.

\section{Conséquences pour l'évaluation comparée de l'office des procédures}

L'étude comparée des procédures de licenciement montre à quel point des simplifications, telle que l'association univoque et causale entre l'existence d'une règle expresse et la complexité de la procédure, doivent être évitées. La lecture procédurale proposée ici est basée sur un principe simple: celui d'identifier les procédures applicables à une situation de licenciement. Ce principe demande que soient clarifiées les procédures propres à chaque ordonnancement juridique national, de même que 
la nature et la structure temporelle des processus de licenciement. Toute évaluation de l'efficience comparée de ces procédures nécessite donc la construction d'outils plus subtils que ceux utilisés actuellement dans les comparaisons internationales menées par l'OCDE, la Banque mondiale, mais également par la Commission européenne pour l'efficacité de la justice (CEPEJ) qui propose des taux de contestation des décisions de licenciement dans les pays membres du Conseil de l'Europe.

En dépit du fait qu'ils proclament prendre en compte, avec plus ou moins de précautions méthodologiques selon qu'il s'agît de l'indicateur LPE ou de l'indicateur $E W$, les règles applicables, ils sont très peu procéduraux. Ou, plus exactement, les dimensions procédurales sont en fait transformées ipso facto en dimensions substantielles de la protection de l'emploi. Le fait que l'OCDE confère au droit du travail la fonction (au demeurant légitime) de "protéger les emplois» est à cet égard très significatif: or, la logique procédurale des licenciements ne se résume pas à protéger, de manière quelque peu illusoire, des emplois ou des contrats.

Ensuite, les évaluations en terme de durées des procédures, outre qu'elles doivent prendre en compte l'intégralité des étapes procédurales, ne doivent pas postuler que les procédures les plus courtes sont les plus efficaces.

De plus, les études des taux de recours, outre qu'ils ne peuvent uniquement considérer les contentieux judiciaires, doivent prendre en considération qu'une rupture du contrat de travail est par nature source de litige, et qu'il n'est pas forcément malsain ou anormal qu'un contentieux apparaisse et soit tranché, de manière judiciaire ou extrajudiciaire. De plus, une discordance marquée caractérise les résultats de l'OCDE et du CEPEJ sur les taux de recours judiciaire dans différents pays. Par exemple, l'Italie est considérée par le CEPEJ (CEPEJ, rapport 2006) comme un pays ayant un taux élevé de recours contre les licenciements (276 affaires nouvelles pour 100000 habitants) (CEPEJ, 2006, p. 94), qui surpasse celui de la France (203 affaires nouvelles pour 100000 habitants) et du Royaume-Uni (160 affaires pour 100000). Inversement, 1'OCDE (Perspectives de l'emploi, 2004, pp. 74-75) conclut que l'Italie est un pays faiblement contentieux: le nombre total de litiges soumis aux tribunaux ne serait que de $3864(1,6 \%$ des licenciements en 2001), contre 92000 affaires litigieuses en France $(25,3 \%$ des licenciements en $2001(2))$ et 42000 au Royaume-Uni (7,1\% des licenciements).

(2) En réalité ce taux est celui des licenciements pour motif personnel. Le taux de contestation des licenciements économiques, individuels ou collectifs, est de l'ordre de $2,5 \%$. Or, le rapport de l'OCDE consacré aux perspectives de l'emploi 2004 ne donne pas cette précision. Sur ce point, voir SERVERIN et al., 2008a
Enfin, on observera, à l'aide des données de l'OCDE, que le taux de contrats à durée indéterminée par rapport à l'emploi salarié reste remarquablement stable en France (entre 84 et $86 \%$ entre 2000 et 2007) et au Royaume-Uni (93 ou 94\% sur la même période) et progresse légèrement en Italie (de $56 \%$ en 2000 à $61 \%$ en 2007). Les données disponibles sur les États-Unis ne forment pas une série complète sur la période, mais montrent que le taux de contrats «permanents» est identique en 2001 et en 2005: 96\%). Quant au climat social, les données du BIT montrent que le nombre de grèves et de lock-out, ainsi que le nombre de journées de travail non effectuées pour 1000 salariés, sont dans les quatre pays soit stables à un niveau faible (ÉtatsUnis), soit en tendance déclinante, à l'exception du Royaume-Uni entre 2005 et 2007. Ces données descriptives laissent à penser que l'encadrement formel des procédures de licenciements est un bon outil de prévention des litiges du travail et, plus généralement, des conflits sociaux. Ce jugement mériterait cependant d'être confirmé empiriquement.

\section{Mesurer les règles: périmètre, jurisprudence et conventions collectives}

La discussion des indicateurs leximétriques est souvent formulée en termes de mesures de jure ou de facto, respectivement associées aux «règles formelles» (expression de Botero et al., 2004) et, selon les cas, à leur enforcement, à «la pratique», aux coûts économiques réels de l'observation des règles, ou encore «ce qui est supposé être mis en œuvre». Les glissements sémantiques quant au contenu des mesures de facto sont un signe du malaise qui caractérise les approches en termes d'indicateurs leximétriques. Au sens strict, la notion anglo-américaine d'enforcement renvoie à la garantie judiciaire des droits, mais ce n'est pas dans ce sens que les usages économiques lui attribuent; les «coûts économiques» sont définis soit par des règles législatives ou réglementaires, soit par des conventions collectives, qui sont dans tous les cas des règles «formelles»; enfin, la notion de «ce qui est supposé être mis en œuvre» illustre à merveille un propos allusif.

À cette distinction commode mais infructueuse et difficile à manier entre de jure et de facto, nous substituons une autre grille de lecture, centrée sur la définition des normes, leur champ d'application et le sens empirique que leur donnent les tribunaux via le contentieux et la jurisprudence. L'approche que nous privilégions consiste à considérer que les règles sont des supports effectifs des actions, et que 
par conséquent le mode d'existence des règles passe par les procédures et leur administration(3).

Plutôt que de procéder à un examen critique de l'ensemble des problèmes que pose la méthode des indicateurs, nous limiterons notre propos à la capacité des indicateurs LPE de l'OCDE et $E W$ de Doing Business à capturer la réglementation de l'emploi autrement qu'en termes nominaux. Plutôt que d'une discussion en termes de de jure ou de facto, qui est le cadre usuel de discussion des indicateurs, nous nous intéressons aux dispositifs procéduraux effectivement applicables à la rupture du contrat de travail dans le cadre de licenciements individuels ou collectifs. Trois points seront successivement abordés: le périmètre de l'emploi salarié saisi par les indicateurs; la mise en œuvre des droits dans le contentieux et l'interprétation des dispositifs légaux par la jurisprudence; les couvertures conventionnelles et les dispositifs mis en œuvre par les entreprises sans qu'ils relèvent d'une mesure législative ou réglementaire expresse.

\section{Périmètre et représentativité}

Sur ce point, les indicateurs de $D B$ et de l'OCDE apparaissent sensiblement différents.

Pour la construction de l'indicateur $E W$ de $D B$, le recueil des données est basé sur un cas particulier: un «salarié standardisé» qui présente des caractéristiques précises énumérées dans les instructions adressées aux répondants aux enquêtes: un salarié à temps plein, père de deux enfants et dont l'épouse est au foyer, d'une ancienneté de vingt ans au sein de la même entreprise, dont le salaire et les prestations sociales sont égaux au ratio $\mathrm{PNB}$ /population active du pays au cours de la totalité de sa période de travail, qui est citoyen, de la race et de la religion majoritaire dans le pays, et n'est pas syndiqué (à moins que l'appartenance syndicale ne soit obligatoire). Ce «salarié standardisé» est lui-même employé dans une «entreprise standardisée» dont les caractéristiques sont elles aussi précisément énumérées: une entreprise locale (sans participation étrangère) du secteur manufacturier de plus de 200 salariés, qui n'accorde aucun avantage salarial qui ne soit prévu par les lois et règlements. BERG et CAZEs (2007) voient dans ce procédé la source d'un biais de sélection. En effet, Doing Business ne justifie pas le choix de l'entreprise de 200 salariés et ne précise pas en quoi elle est représentative des salariés dans les différents pays. Ainsi, dans l'Union européenne à quinze en 2005 , l'ancienneté moyenne était de 10,6 ans, et $17 \%$ des salariés seulement avaient une ancienneté supérieure à 20 ans. De surcroît, l'indicateur $D B$ ne tient aucunement compte de l'existence de différentiels de

(3) Ce point de vue est étoffé dans Serverin et al., 2008a et 2008b. niveau de protection de l'emploi en fonction de la taille des entreprises. En Allemagne par exemple, les entreprises de moins de cinq salariés, qui ne sont pas soumises au Kündigungsschutzgesetz (loi de protection contre les licenciements), emploient $11 \%$ des salariés allemands. Au Chili, $60 \%$ des salariés sont employés dans des entreprises dont l'effectif est inférieur à cinquante (BERG, CAZES, 2007).

L'indicateur synthétique de LPE de l'OCDE ne s'appuie pas sur une situation de référence concernant les caractéristiques de l'entreprise ou du salarié. Parmi les dix-huit indicateurs élémentaires, certains envisagent plusieurs situations d'ancienneté des salariés ou d'effectifs de salariés licenciés. Cependant, la taille des établissements ou des entreprises n'est pas prise en compte dans la définition du score attribué aux indicateurs élémentaires. $\mathrm{Ne}$ sont pas prises en compte, ou le sont de manière sous-évaluée, les dispositions conventionnelles relatives notamment aux préavis et aux indemnités de licenciement.

\section{Un double angle mort: le contentieux et la jurisprudence}

La question de savoir ce que les indicateurs mesurent (et ne mesurent pas) a été posée à de nombreuses reprises. BERG et CAZES estiment à cet égard que "The $(D B)$ index is based on a partial and crude understanding of how labour markets and its institutions function as well as the purpose of labour law" (BERG, CAZES, 2007, p. 1). Nous laisserons ici de côté la question de l'objet du droit du travail pour nous en tenir au premier aspect de la citation précédente.

On s'interroge ici sur l'information capturée et non capturée par les indicateurs qui sont sensés, du point de vue de leurs producteurs et de leurs utilisateurs, délivrer une information objective sur la nature et l'intensité des contraintes réglementaires qui pèseraient sur les acteurs économiques, les employeurs plus particulièrement. Les auteurs qui critiquent ces indicateurs se fondent sur une distinction entre ce qui est de jure et de facto, en reprochant aux indicateurs de ne désigner que le droit «formel», par rapport au droit «dans les faits». C'est le cas de KaUfMAn et al. (2003), et des critiques de Botero et al. (2004), dont la réponse fait écho à la même distinction. Il est cependant difficile de savoir en quoi consiste la description du droit «factuel», de sorte que l'objet de la critique reste ambigu. Ou bien le factuel désigne les conduites réelles des sujets par rapport aux commandements édictés par les règles, ou bien il s'agit des règles sanctionnées par les juges (Enforcement), ou bien il s'agit de l'interprétation qu'en donnent les tribunaux. La première définition renvoie au suivi des règles; les deux autres renvoient à l'intervention des tribunaux, sous leur double dimension contentieuse et jurisprudentielle. 
La contribution des tribunaux à la mise en œuvre du droit du travail, donc à la réalisation effective de la protection de l'emploi, constitue un obstacle sur lequel butent les indicateurs LPE et $E W$.

\section{Un point commun aux indicateurs LPE et EW}

L'OCDE admet explicitement cette limite de l'indicateur de LPE : les dispositions légales en matière de protection de l'emploi «font l'objet d'interprétations de la part des tribunaux, ce qui peut constituer une source importante (mais difficile à cerner) de variation de la rigueur de la LPE, à la fois entre certains pays et dans le temps » (OCDE, Perspectives de l'emploi 2004, p. 71).

Quant à l'indicateur $E W$, certains commentateurs ont souligné qu'il se limite à l'évaluation des textes, ne se préoccupe pas de leur «application» et omet une variable Enforcement Procedures, Or, "Enforcement plays a crucial role in the functioning of labour markets, notably in determining labour market flows such as job losses and inflows into unemployment"; la conséquence étant que "Jurisprudence would thus be seen as important as - if not more important than - the nominal strictness of regulations per se" (BERG, CAZES, 2007, p. 10).

\section{Deux lectures du rôle des tribunaux: jurisprudence et contentieux}

L'intuition selon laquelle l'observation de la protection effective de l'emploi implique la prise en compte de l'action des tribunaux doit être précisée. Cette intervention est de deux ordres, ou bien d'ordre interprétatif (jurisprudence), ou bien d'ordre juridictionnel (contentieux). L'action jurisprudentielle renvoie à la prise en considération de l'interprétation des règles législatives ou réglementaires par les tribunaux supérieurs. Le contentieux recouvre les actions en justice, ainsi que les réponses données par les juges (réussite ou échec de l'action).

Une lecture jurisprudentielle de la mise en œuvre du droit du travail peut conduire à prendre en considération les interprétations de la réglementation par les tribunaux supérieurs, détenteurs du pouvoir de fixer le sens des règles législatives ou réglementaires. À ce titre, les positions jurisprudentielles participent du droit positif et s'attachent à la formation des normes juridiques, mais elles ne peuvent par nature décrire la nature du contentieux du travail, qui doit faire l'objet d'une autre méthode. La lecture en termes de contentieux invite à rechercher quelles demandes sont formées par les salariés parmi les demandes possibles, et quelles réponses leur sont apportées par les tribunaux.

On peut noter que certains travaux se sont efforcés d'intégrer dans leurs modèles les positions des juges du fond en matière de conflit du travail, en utilisant les statistiques judiciaires. Ainsi, Marinescu (2003, 2006) s'est attachée à analyser la relation empi- rique entre l'application du droit du licenciement par les tribunaux en France et au Royaume-Uni et la conjoncture économique. Elle souligne que l'adaptabilité constatée des décisions des tribunaux aux circonstances conjoncturelles devrait conduire à nuancer l'idée selon laquelle le système de protection de l'emploi est particulièrement rigide et peu sensible à ses conséquences économiques. Dans le même cadre analytique, ICHINO, PoLo et RETTORE (2003) ont procédé à une étude empirique du contentieux du licenciement en Italie. Ils concluent que les juges italiens sont sensibles aux conditions macro-économiques, dans la mesure où ils tendent à statuer plus fréquemment en faveur du salarié lorsque le taux de chômage régional est élevé, comparativement à une conjoncture moins déprimée. Ces approches empiriques mettent en évidence le fait que la mesure des coûts du licenciement ne peut être valablement opérée à partir des normes légales en vigueur, mais suppose de prendre en considération la manière dont les juges décident en pratique. Or cet aspect n'est pas capturé par les indicateurs de protection de l'emploi.

\section{Couvertures conventionnelles}

Nous nous en tenons ici à l'indicateur de LPE de l'OCDE compte tenu du fait que les précisions méthodologiques données par cette organisation internationale sont suffisamment précises pour en saisir le contenu, en particulier en ce qui concerne la prise en considération des dispositions conventionnelles et leur poids dans la valeur de certaines des variables élémentaires (voir $A$ detailed description of EPL in force in 2003). Cet état de fait n'est pas de mise pour l'indicateur EW de Doing Business, qui, en plus de mesurer les écarts à une situation de référence implicitement normative de licenciement at will, ignore les relations collectives de travail (KIRAT, 2006).

Les commentaires apportés aux scores d'un certain nombre de pays dans les domaines des durées de préavis et du quantum des indemnités de licenciement pour les licenciements individuels montrent que les protections conventionnelles sont assez nettement minorées. La sous-estimation récurrente des valeurs des scores sur ces deux variables caractérise plutôt les pays de common law, et joue par conséquent dans le sens de la surpondération relative des pays où les règles sous plus fréquemment légales que conventionnelles. Ainsi, la Nouvelle-Zélande obtient, pour les trois hypothèses d'ancienneté des salariés licenciés, un score égal à zéro pour les indemnités de licenciement, alors même que la note technique de l'OCDE précise que $21 \%$ des salariés bénéficient d'accords conventionnels qui garantissent de telles indemnités «dans la plupart des cas»; le score nul devrait refléter l'absence totale de mécanisme d'indemnisation de la rupture du contrat de travail à la charge de l'employeur. Outre la Nouvelle-Zélande, le cas des États-Unis ne peut que susciter l'étonne- 
ment: un score égal à zéro pour le régime d'indemnisation des licenciements individuels, en dépit du fait, précisé dans les commentaires des données, qu'ils «puissent faire l'objet dans les accords collectifs d'entreprises». Selon le Labor Department, environ $20 \%$ des salariés du secteur privé bénéficiaient, en 2000 , de dispositifs d'indemnisation du licenciement, ce qui ajoute au trouble sur la valeur du score de zéro.

Si l'on considère les dispositifs relatifs aux conséquences de licenciements non justifiés (Unjustified Dismissal), notamment celles qui concernent l'éventuelle réintégration des salariés concernés, la note technique de l'OCDE distingue, dans les commentaires du tableau déjà cité, entre l'existence d'un dispositif légal et son niveau, ou sa fréquence, d'utilisation. On peut en passant se poser la question des sources qui autorisent à qualifier une fréquence d'activation d'une disposition légale, point qui n'est précisé ni dans la note technique, ni dans les Perspectives de l'emploi. Cela étant dit, on pourrait s'attendre que les pays qui présentent ces caractéristiques - un dispositif légal peu activé - soient l'objet de scores proches, sinon égaux, à cet égard. Or, il n'en n'est rien. À titre d'exemples de pays partageant cette caractéristique, l'Autriche, l'Allemagne et le Danemark ont des scores égaux respectivement à $3,1,5$ et 1 . Le cas de l'Irlande, noté 1 , où la réintégration est «possible», est source d'étonnement, de même que celui de l'Espagne, noté zéro, dans la mesure où les salariés ont le choix entre demander leur réintégration ou une indemnisation de leur licenciement «injustifié. Enfin, pour finir, en Nouvelle-Zélande, l'Employment Relations Act exige de l'Employment Relations Authority de prononcer la réintégration Whenever Practicable», ce qui s'accompagne d'une note de 1 .

En définitive, les valeurs de notes pour les variables élémentaires ou des sous-indices ne sont pas en réelle concordance avec la description des dispositifs légaux et, surtout, conventionnels. Tout comme pour la jurisprudence, le rapport Perspectives de l'emploi 2004 se limite à préciser, de manière significativement laconique, que, à l'exception du Japon, «dans la plupart des autres pays, il est difficile de prendre en considération les contrats individuels ou les conventions collectives prévoyant des indemnités de licenciement» (OCDE, 2004, encadré 2.1., p. 72).
Faute de construction d'une méthode comparative capable de construire les équivalents fonctionnels, il est difficile d'identifier les règles pertinentes applicables à la rupture du contrat de travail, soit qu'elles opèrent de manière discrète (sous une forme autre que celle d'une règle figurant expressément dans un texte étatique), soit qu'elles ne relèvent pas d'un régime juridique spécial, mais du droit commun des obligations ou des droits fondamentaux. Une des exigences du droit comparé est à cet égard de ne pas s'en tenir à la forme et au libellé des règles, mais de repérer les «équivalents fonctionnels».

Il s'avère que les classements internationaux fondés sur des mesures de la réglementation ne manquent pas d'appeler des réserves, plus particulièrement au regard du bornage de la séquence et des règles observées en matière de pratique du licenciement. L'évaluation de la réglementation peut, dans l'absolu, procéder de deux points de départ: soit celui des règles de droit positif, ce qui revient à entrer par les normes expressément applicables au processus de licenciement; soit celui des situations de gestion de la rupture du contrat du travail. Partir des règles de droit positif pose deux risques: a) déduire de l'énoncé des règles une description des situations réelles (ce qui ne veut pas pour autant confirmer l'idée d'ineffectivité); b) considérer que internationalement les situations sont régies par des règles équivalentes (de même objet, avec la même structure du processus, abstraites, générales et systématisables), dont on peut évaluer et mesurer la rigueur. Ce choix méthodologique a pour effet d'éluder les situations effectives dont la description doit tenir compte: a) de la diversité des règles de licenciement en fonction de la taille de l'entreprise, du droit national, de la couverture conventionnelle, voire du système de relations professionnelles; b) de la diversité des formes de présence du droit (soit de façon directe, dans les pays de droit civil, soit par des médiations du type procédure de griefs dans les pays de common law). 


\section{Bibliographie}

Ahlering B., Deakin S.(2005) «Labour regulation, corporate governance and legal origin : a case of institutional complementarity?», Law and Society Review, Vol. 41, $\mathrm{N}^{\circ}$ 4, pp. 865-908.

Arruñada B. (2007), «Pitfalls to avoid when measuring institutions: Is Doing Business damaging business?», Journal of Comparative Economics, 35, pp. 729-747.

Berg J., Cazes S. (2007), «The Doing Business Indicators: Measurement issues and political implications», ILO - Economic and Labour Market Analysis Department, Economic and Labour Market Paper 2007/6.

Bertola G., Boeri T., Cazes S. (2000), «Employment protection in industrialized countries: the case for new indicators», International Labour Review, 139 (1).

BIT (2007), «Rapport de la Banque mondiale sur la pratique des affaires (Doing Business): l'indicateur d'embauche des travailleurs», $300^{\mathrm{e}}$ session du conseil d'administration, Genève, novembre 2007 (document BG. 300/4/1).

Boвbio M. (2008), «De la difficulté de licencier...», in Meda D. et Serverin E. (sous la direction de), Le contrat de travail, collection «Repères», Paris, La Découverte.

Botero J., Djankov R., La Porta F., Lopez-De-Silanes A., Shleifer A. (2004), "The Regulation of Labor», Quarterly Journal of Economics, 119 (4), pp. 1339-1382.

Cahuc P., Kramarz F. (2004), De la précarité à la mobilité: vers une Sécurité sociale professionnelle, rapport au ministre d'État, ministre de l'économie des finances et de l'industrie, et au ministre de l'emploi du travail et de la cohésion sociale, collection «Rapports officiels», Paris, La Documentation française.

Camerlinck C. (1982), Traité de droit du travail, tome I, Le contrat de travail, Paris, Dalloz.

CEPEJ (2006), Systèmes judiciaires européens.

DALmasso R. (2009), Essai sur le concept de licenciement économique, étude comparée des droits français et italiens, collection «Bibliothèque de droit Social», t. 49, Paris, LGDJ.

Deakin S., Lele P., Siems M. (2007), «L'évolution du droit du travail: évaluation et comparaison des régimes réglementaires», Revue internationale du travail, vol. 146, n 3-4, pp. 143-178.

Edelman L. B., Uggen C, Erlanger H.S. (1999), “The Endogeneity of Legal Regulations: Grievance Procedures as Rational Myth", American Journal of Sociology, Vol. 105, N² 2, sept., pp. 406-454.

Edelman L. B., Krieger L.H., Eliason S.R., Albiston C, Mellema V. (2008), "When Organizations Rule: Judicial Defence to Institutionalized Employment Structures", draft, University of California-Berkeley.
Ichino A., Polo M., Rettore E. (2003), “Are judges biased by labor market conditions?", European Economic Review, 47, pp. 913-944.

KIRAT T. (2006), «Les indicateurs de protection de l'emploi : la mesure du droit du travail en question?», in Kirat T., Valentin J., (sous la direction de), «La protection de l'emploi en question», Économie et Institutions, $\mathrm{n}^{\circ} 9$, pp. 85-127.

LAMBERT E. (1921), Le gouvernement des juges et la lutte contre la législation sociale aux États-Unis. L'expérience américaine de contrôle judiciaire de la constitutionnalité des lois, Giard et Brière (réédition 2005, Paris, Dalloz).

Marinescu I. (2003), «Application du droit du licenciement et conditions économiques en France», document de travail.

MARINESCU I. (2006), «Are Judges Sensitive to Economic Conditions? Evidence from UK Employment Tribunals», document de travail.

Markesinis B. S. (1997), Foreign Law and Comparative Methodology: A Subject and a Thesis, Hart Publication.

OCDE (2004) A detailed description of employment protection regulation in force in 2003, Paris, OCDE.

Ocde (2005), Perspectives de l'emploi 2004, Paris, OCDE.

Oyer P., Schaefer S. (2000), "Layoffs and Litigation", Rand Journal of Economics, 31 (2), pp. 345-358.

SERVERIn E. (2007), «Le traitement des litiges du travail en droit français et britannique: deux modèles d'intégration des procédés amiables » Revue de droit du travail $\mathrm{n}^{\circ} 4$, avril.

Serverin E., Valentin J., Kirat T, Sauze D., Dalmasso R. (2008a), «L'évaluation du droit du travail à la lumière de son contentieux : comparaison des droits et des procédures, mesure des actions», in: L'évaluation $d u$ droit du travail: problèmes et méthodes, Institut international pour les études comparatives - DARES (Appels d'offres L'évaluation économique du droit du travail), février 2008.

Serverin E., Valentin J. Kirat T, Sauze D., Dalmasso R. (2008b), «Évaluer le droit du licenciement. Comparaison des droits et des procédures, mesure des actions », Revue de l'OFCE, $\mathrm{n}^{\circ}$ 107, oct., pp. 1-36.

Siems M. (2005), "Numerical Comparative Law: Do We Need Statistical Evidence in Order to Reduce Complexity?", Cardozo Journal of International and Comparative Law, 13, pp. 521-540.

Zweigert K., Kötz, H. (1998), Introduction to comparative law, 3rd Revised Edition, translated from the German by Tony Weir, Clarendon Press, Oxford. 


\section{Tableau A : Licenciement individuel}

Ce tableau doit être lu de haut en bas. Il retrace chronologiquement les étapes procédurales des licenciements individuels des trois pays étudiés La rupture du contrat n'est jamais la première étape procédurale devant être menée par l'employeur, ni la dernière. Les étapes mentionnées constituent des situations procédurales; leurs durées ne sont pas équivalentes.

\begin{tabular}{|c|c|c|}
\hline France & Royaume-Uni & Italie \\
\hline \multicolumn{3}{|l|}{$\begin{array}{l}\text { Obligation permanente d'adaptation et de } \\
\text { formation (en matière de licenciement pour } \\
\text { motif économique). Article L. 1233-4 du } \\
\text { Code du travail }\end{array}$} \\
\hline & $\begin{array}{l}\text { Procédure de règlement des litiges sur le lieu } \\
\text { de travail. Plusieurs étapes à suivre: Written } \\
\text { Statement (déclaration écrite); Meeting } \\
\text { (entrevue); décision de l'employeur; Appeal } \\
\text { Meeting (entrevue d'appel). Employment Act } \\
\text { (Dispute Resolution) 2002, entré en vigueur } \\
\text { en } 2004\end{array}$ & \\
\hline \multicolumn{3}{|c|}{$\begin{array}{l}\text { Procédures conventionnelles préalables aux } \\
\text { procédures légales de licenciement (rare mais } \\
\text { possible) } \\
\text { Procédure individuelle de licenciement: } \\
\text { convocation à un entretien préalable; entre- } \\
\text { tien préalable; délai de réflexion. Articles } \\
\text { L. } 1232-2 \text { à L. } 1232-5 \text { (licenciement pour } \\
\text { motif personnel); L. } 1233-11 \text { à L. } 1233-14 \\
\text { (licenciement pour motif économique }\end{array}$} \\
\hline $\begin{array}{l}\text { Notification écrite et motivée de la rupture. } \\
\text { Articles L. 1232-6; L. 1233-15 }\end{array}$ & Notification écrite et motivée de la rupture & $\begin{array}{l}\text { Notification écrite de la rupture. Le } \\
\text { salarié peut demander communication } \\
\text { par écrit les motifs justifiant son licencie- } \\
\text { ment. Article } 2 \text { de la loi }{ }^{\circ} 604 / 1966 \text { du } \\
15 \text { juillet } 1966\end{array}$ \\
\hline $\begin{array}{l}\text { Rupture du contrat } \\
\text { Existence d'un préavis sauf exception } \\
\text { Versement d'indemnités de licenciement }\end{array}$ & $\begin{array}{l}\text { Rupture du contrat } \\
\text { Existence d'indemnités légales de licencie- } \\
\text { ment en matière de licenciement économique }\end{array}$ & $\begin{array}{l}\text { Rupture du contrat } \\
\text { Existence d'un préavis sauf exception } \\
\text { Traitement de fin de rapport (pour tout } \\
\text { licenciement) } \\
\text { En cas de licenciement «économique», } \\
\text { versement d'une indemnité de mobilité et } \\
\text { inscription sur liste de mobilité }\end{array}$ \\
\hline $\begin{array}{l}\text { Prescription de l'action portant sur la régu- } \\
\text { larité ou la validité du licenciement: douze } \\
\text { mois à compter de la notification du licencie- } \\
\text { ment économique. Article L. } 1235-7\end{array}$ & $\begin{array}{l}\text { Pour avoir le droit de contester le licencie- } \\
\text { ment, il faut, pour le salarié, une ancienneté } \\
\text { d'un an } \\
\text { La contestation d'un licenciement se prescrit } \\
\text { dans un délai de trois mois à compter de la } \\
\text { rupture }\end{array}$ & $\begin{array}{l}\text { Contestation possible du licenciement } \\
\text { dans un délai de soixante jours à compter } \\
\text { de la notification du licenciement }\end{array}$ \\
\hline $\begin{array}{l}\text { Aucune tentative obligatoire de conciliation } \\
\text { extrajudiciaire } \\
\text { Transaction possible (et fréquente) } \\
\text { Arbitrage possible }\end{array}$ & $\begin{array}{l}\text { Saisine du tribunal, qui sursoit à statuer dans } \\
\text { l'attente de la conciliation } \\
\text { Tentative obligatoire de conciliation devant un } \\
\text { organisme indépendant (ACAS) } \\
\text { En cas d'échec de la conciliation, le tribunal } \\
\text { se trouve pleinement saisi pour statuer sur le } \\
\text { litige } \\
\text { Transaction et arbitrage possibles }\end{array}$ & $\begin{array}{l}\text { Tentative obligatoire de conciliation } \\
\text { extrajudiciaire devant une commission } \\
\text { de conciliation instaurée par l'adminis- } \\
\text { tration du travail, ou devant une commis- } \\
\text { sion de type syndical. Articles } 410 \text { et } \\
\text { suivants du Code de procédure civile } \\
\text { En cas d'échec de la conciliation, saisine } \\
\text { du tribunal possible } \\
\text { Transaction possible mais encadrée. } \\
\text { Arbitrage possible }\end{array}$ \\
\hline $\begin{array}{l}\text { Saisine du conseil de prud'hommes } \\
\text { Tentative obligatoire de conciliation devant le } \\
\text { bureau de conciliation. Article L. 1411-1 }\end{array}$ & $\begin{array}{l}\text { Saisine de l'Employment Tribunal en cas } \\
\text { d'échec de la conciliation (compétence rési- } \\
\text { duelle des County Courts) }\end{array}$ & $\begin{array}{l}\text { Saisine du Tribunale en cas d'échec de la } \\
\text { conciliation. Ultime tentative de concilia- } \\
\text { tion lors de l'audience }\end{array}$ \\
\hline
\end{tabular}


En cas de licenciement sans cause réelle et sérieuse (ou de licenciement abusif), prédominance d'une logique indemnitaire (la rupture demeure)

Rares possibilités de nullité du licenciement avec réintégration
En cas de licenciement Unfair, large latitude du juge. Il peut imposer la réintégration (rare), ou indemniser la victime (hypothèse la plus fréquente) (Employment Right Act 1996, point $\left.n^{\circ} 114\right)$
Deux régimes coexistent. Protection réelle (pour les grandes entreprises): principe de la réintégration du salarié avec octroi de dommages et intérêts Protection obligatoire (pour les petites entreprises) réembauche du salarié, ou, au choix de l'employeur, indemnisation du salarié

Article 18 loi no 300/1970 (statut des travailleurs) du 20 mai 1970; article 8 loi $n^{\circ} 604 / 1966$ modifié par loi n $108 / 1990$

Tableau B : Licenciements collectifs (étapes supplémentaires par rapport à celles indiquées dans le tableau 1)

Les étapes mentionnées complètent, ou parfois se substituent, à celles mentionnées au tableau 1.

\begin{tabular}{|c|c|c|}
\hline France & Royaume-Uni & Italie \\
\hline $\begin{array}{l}\text { Obligation d'adaptation et de formation } \\
\text { Fixation (facultative) conventionnelle de } \\
\text { critères d'ordre des licenciements. Existence, } \\
\text { à défaut d'accord, de critères légaux. Article } \\
\text { L. 1233-5 } \\
\text { Accord «de méthode» (facultatif) sur } \\
\text { la procédure de licenciement. Articles } \\
\text { L. 1233-21 et suivants }\end{array}$ & $\begin{array}{l}\text { Possibilité de fixer de manière préventive les critères } \\
\text { de sélection des salariés à licencier en priorité }\end{array}$ & $\begin{array}{l}\text { Possibilité d'encourager la fixation } \\
\text { conventionnelle avec les repré- } \\
\text { sentants des salariés les critères } \\
\text { de choix des salariés à licencier. } \\
\text { À défaut d'accord, existence de } \\
\text { critères légaux en concours entre } \\
\text { eux. Article } 5 \text { de la loi n }{ }^{\circ} 223 / 1991 \\
\text { du } 23 \text { juillet } 1991\end{array}$ \\
\hline $\begin{array}{l}\text { Procédure des grands licenciements collec- } \\
\text { tifs (dix salariés et plus sur trente jours): } \\
\text { double information et consultation du comité } \\
\text { d'entreprise (prérogatives économiques géné- } \\
\text { rales; plan de sauvegarde de l'emploi) } \\
\text { Notification du projet à l'administration } \\
\text { compétente, qui peut émettre un avis sur la } \\
\text { procédure } \\
\text { Pour les petits licenciements collectifs, infor- } \\
\text { mation et consultation allégée des institutions } \\
\text { représentatives du personnel } \\
\text { Prise en compte d'une obligation de } \\
\text { reclassement }\end{array}$ & $\begin{array}{l}\text { Procédure de Redundancy (licenciement des salariés } \\
\text { en sureffectif) si vingt postes ou plus sont concernés. } \\
\text { L'employeur doit établir un plan permettant de } \\
\text { déterminer le nombre et le type de salariés en suref- } \\
\text { fectif. Information et consultation des représentants } \\
\text { des salariés (Workforce), qui peuvent proposer des } \\
\text { solutions alternatives. Délais de procédure de } 30 \text { à } \\
90 \text { jours } \\
\text { Information préalable obligatoire de l'administration } \\
\text { du travail (DTI) } \\
\text { Fondements légaux: } \\
\text { - Information and consultation of employees regula- } \\
\text { tions (2004) statutory instrument } 2004 \mathrm{~N}^{\circ} 3426 \\
\text { - Part IV of the trade union and labour relations } \\
\text { (consolidation) act } 1992 \text { amendé par les textes } \\
\text { suivants: Trade union reform and employment rights } \\
\text { act } 1993 \text {; Collective redundancies and transfert of } \\
\text { undertakings (protection of employment) (amende- } \\
\text { ment) régulation } 1995 \text { (si } 1995 \mathrm{~N}^{\circ} 2587 \text { ); Collective } \\
\text { redundancies and transfert of undertakings (protec- } \\
\text { tion of employment) (amendment) regulation } 1999 \\
\text { (si } 1999 \mathrm{n}^{\circ} 1925 \text { ); Collective redundancies (amend- } \\
\text { ment) regulations } 2006 \text { (si } 2006 \mathrm{~N}^{\circ} 2387 \text { ) }\end{array}$ & $\begin{array}{l}\text { Procédure de licenciement collectif } \\
\text { pour réduction de personnel (loi } \\
\mathrm{n}^{\circ} 223 / 1991 \text { du } 23 \text { juillet } 1991 \\
\text { (cinq salariés et plus par unité } \\
\text { productive dans une même période } \\
\text { de } 120 \text { jours). Consultation des } \\
\text { syndicats sur le motif déterminant } \\
\text { la situation, les motifs techniques, } \\
\text { et les raisons des ruptures. Examen } \\
\text { conjoint avec les syndicats (possi- } \\
\text { bilités de propositions alternatives) } \\
\text { et possibilité facultative de parvenir } \\
\text { à un accord (signature d'un contrat } \\
\text { collectif). } \\
\text { Paiement à l'INPS par l'employeur } \\
\text { d'une indemnité de mobilité } \\
\text { (paiement réduit en cas d'accord } \\
\text { avec les syndicats) }\end{array}$ \\
\hline Litiges collectifs éventuels sur la procédure & Litiges collectifs éventuels sur la procédure & $\begin{array}{l}\text { Litiges collectifs éventuels sur la } \\
\text { procédure }\end{array}$ \\
\hline Rupture des contrats & Rupture des contrats & Rupture des contrats \\
\hline
\end{tabular}

